\title{
Effects of temperature on the development and fecundity of Microplitis \\ similis (Hymenoptera: Braconidae), a parasitoid of Spodoptera litura (Lepidoptera: Noctuidae)
}

\section{Si-Jia Yi}

Hunan Provincial Key Laboratory for Biology and Control of Plant Diseases and Insect Pests, Hunan Agricultural University, Changsha, Hunan, P. R. China

Richard J. Hopkins

Natural Resources Institute, University of Greenwich, Chatham Maritime, Kent, UK

Xu-Yang Chen

Hunan Provincial Key Laboratory for Biology and Control of Plant Diseases and Insect Pests, Hunan Agricultural University, Changsha, Hunan, P. R. China

Zhuang-Mei Chen

Hunan Provincial Key Laboratory for Biology and Control of Plant Diseases and Insect Pests, Hunan Agricultural University, Changsha, Hunan, P. R. China

Xing Wang

Hunan Provincial Key Laboratory for Biology and Control of Plant Diseases and Insect Pests, Hunan Agricultural University, Changsha, Hunan, P. R. China

Guo-Hua Huang

Corresponding Author

E-mail address: ghhuang@hunau.edu.cn

https://orcid.org/0000-0002-6841-0095

Hunan Provincial Key Laboratory for Biology and Control of Plant Diseases and Insect Pests, Hunan Agricultural University, Changsha, Hunan, P. R. China 
Correspondence: Guo-Hua Huang, Hunan Provincial Key Laboratory for Biology and Control of Plant Diseases and Insect Pests, Hunan Agricultural University, Nongda Road 1, Furong District, Changsha, Hunan 410128, P. R. China.

\section{Abstract}

Microplitis similis (Hymenoptera: Braconidae) is a solitary endoparasitoid of Spodoptera litura larvae (Lepidoptera: Noctuidae). Here, the effects of constant temperature $\left(18,21,24,27,30,33\right.$ and $\left.36^{\circ} \mathrm{C}\right)$ on the development and fecundity of $M$. similis developing in S. litura were studied in the laboratory to clarify the range of its potential distribution and better understand its potential as a biological control agent. The developmental duration of $M$. similis varied from $10.6\left(33{ }^{\circ} \mathrm{C}\right)$ to 27.9 days $\left(18^{\circ} \mathrm{C}\right)$. The developmental threshold temperature and effective accumulative temperature of $M$. similis were $9.96^{\circ} \mathrm{C}$ and 231.14 Degree-days, respectively. The average adult longevity of $M$. similis ranged from $5.1\left(33{ }^{\circ} \mathrm{C}\right)$ to 26.8 days $\left(18^{\circ} \mathrm{C}\right)$. The maximum fecundity of the parasitoid was observed at 27 and $30^{\circ} \mathrm{C}$, which were 43.07 and 39.73 eggs, respectively. The minimum fecundity of the parasitoid was observed at $18{ }^{\circ} \mathrm{C}$, which was 8.27 eggs. The intrinsic rate of increase $\left(r_{m}\right)$ and finite rate of increase $(\lambda)$ of $M$. similis were the highest at $30^{\circ} \mathrm{C}$. The net reproduction rate $\left(R_{0}\right)$ was the highest at $27^{\circ} \mathrm{C}$ and $30^{\circ} \mathrm{C}$, which were 44.34 and 40.39 , respectively. We concluded that temperatures in the range 27$30{ }^{\circ} \mathrm{C}$ are the most suitable for development and reproduction of M. similis. Our study provides detailed basic information for development and reproduction of $M$. similis under different temperature conditions.

\section{Introduction}

The oriental leaf worm moth, Spodoptera litura (Fabricius) (Lepidoptera: Noctuidae), is a generalist herbivore and a serious pest in several agricultural cropping systems (Shad et al., 2012). S. litura has been recorded from 109 families and on a total of 389 species of plants, causing serious damage to important crops such as cotton, rice, tomato, tobacco and several vegetables (Tuan et al., 2014; Negi et al., 2016). S. litura, similar to S. exigua larvae, mainly feed on leaves and kill crop plants during extreme outbreaks (Bajpai et al., 2006; Yu et al., 2020a, $\underline{\text { b) }}$. Conventional control of the oriental leaf worm moth has relied on insecticide spraying; however, spraying has led to many problems because of the unreasonable application of insecticides, including environmental pollution, the rapid evolution of insecticide resistance, reduction of natural enemy populations and impacts on human health (Kranthi et al., 2002; Su et al., 2012).

Integrated pest management (IPM) programmes encourage the contribution of natural enemies to the biological control of crop pests (Zhou et al., 2010; Barzman et al., 2015). Kaas et al. (1993) and Zhou et al. (2010) have demonstrated that parasitoids reduce the populations of S. litura and S. exigua, and it is important 
to understand the range of conditions under which this best operates, particularly as climate change is leading to rising temperatures. Microplitis similis Lyle (Hymenoptera: Braconidae) is a solitary parasitoid, and previous studies have reported that M. similis can parasitize Agrotis ipsilon (Rottemberg), $S$. exigua (Hübner) and S. litura (Fabricius) (Shepard \& Barrion, 1998; Li et al., 2015). Li et al. (2015) showed that M. similis can parasitize S. litura and S. exigua and have significant effects on the food consumption and weight gain of parasitized host larvae. Chen et al. (2017b) reported that $M$. similis can parasitize first to late third instar S. litura larvae and parasitize second and early third instar larvae most heavily, causing parasitized $S$. litura larvae to have a pronounced reduction in food consumption and weight gain. $M$. similis has the potential to significantly suppress $S$. litura population growth and the damage caused by $S$. litura.

Temperature is one of the environmental factors that most significantly influences the development, fecundity and population dynamics of parasitoid wasps (Chen et al., 2015). Temperature fluctuation has a huge effect on parasitoid distribution and abundance in the field, and the effect of temperature on the parasitoids can provide much important information when predicting parasitoid occurrence (Bernal \& Gonzalez, 1993). It is important to determine the suitable temperature range for $M$. similis and thus the limits of its potential distribution. In this study, we describe the development, longevity and fecundity of $M$. similis under five constant temperature regimens under laboratory conditions. This research makes an important contribution to understanding the potential role of M. similis in S. litura biological control.

\section{Materials and methods}

\section{Insect culture}

The colonies of $M$. similis wasps and S. litura larvae were established in the Hunan Provincial Key Laboratory for Biology and Control of Plant Diseases and Insect Pests, Hunan Agricultural University, Changsha, Hunan, China in May 2013. S. litura larvae were reared on pinto bean-based diets until pre-pupation (Li et al., 2015), and second and third instar S. litura larvae were used for these experiments. The colony of $M$. similis was maintained on second and early third instar S. litura larvae. Adults of M. similis were reared in test tubes $(10.0 \mathrm{~cm}$ length $\times 2.0 \mathrm{~cm}$ diameter) with a $30 \%$ honey solution as food, and adult $S$. litura were reared in plastic boxes $(22.0 \times 14.0 \times 9.0 \mathrm{~cm})$ with the same food. Both hosts and parasitoids were maintained in a light incubator (product model: GZX150BSH-xes Shanghai Xinmiao Medical Equipment Manufacturing Co., Ltd, Shanghai, China) at $30 \pm 1{ }^{\circ} \mathrm{C}, 60 \pm 5 \% \mathrm{RH}$ and a photoperiod of 14: 10 (L: D). 


\section{Effect of temperature on development, adult longevity, survival and reproduction of M. similis}

One pair of newly emerged $M$. similis (a female and a male) was placed together for a period of $24 \mathrm{~h}$ to permit mating. Then, the female wasp and $20 \mathrm{~s}$ to early third instar $S$. litura larvae were placed together in a test tube $(10.0 \mathrm{~cm}$ length $\times 2.0 \mathrm{~cm}$ diameter). The test tubes were placed in the light incubators (constant temperature: $18,21,24,27,30,33$ or $36^{\circ} \mathrm{C}$ ), with humidity $50 \pm 10 \% \mathrm{RH}$ and a photoperiod of 14: 10 (L: D), and were only removed for a short period to replace the host larval and honey solution. Every $24 \mathrm{~h}$, the host larvae and honey solution were replaced until the parasitoid died. After exposure to the parasitoid, the host larvae were placed in a plastic box $(4.5 \times 12.0 \times 7.0 \mathrm{~cm})$ and cultured in groups provided with the standard pinto bean-based diet. The larvae were cultured in the light incubators under the same temperature used for their exposure to parasitoids and were checked daily until the parasitoid pupated or the larvae died. If a host larva died before parasitoid cocooning or pupation, it was dissected to ascertain whether it contained parasitoid eggs or larvae. When the pupae emerged, the parasitoids were placed individually in a test tube $(10.0 \mathrm{~cm}$ length $\times 2.0 \mathrm{~cm}$ diameter $)$ containing $30 \%$ honey solution without host larvae. The adults were cultured in seven environment chambers (as described above). The honey solution was changed daily, and the parasitoids were checked daily until they died. The developmental duration of each stage (egg-larvae, pupae and all combined pre-adult); the longevity of adult; and the number of total parasitized larvae, pupae and emerging adults were recorded. Each temperature treatment was performed with 15 pairs of parasitoids (a female and a male) that were randomly selected for rearing and then recording their offspring's developmental duration and longevity.

\section{Life table analysis}

The age-stage, two-sex life table approach was used to analyse the life table parameters of $M$. similis (Chi, 1988). The age-stage-specific fecundity $\left(f_{x j}\right)$ (the daily number of eggs laid by an individual at age $x$ and stage $j$ ), the age-specific fecundity curve $\left(m_{x}\right)$ (daily number of eggs produced per female), the age-specific survival rate $\left(l_{x}\right)$ (the probability that a newly oviposited egg will survive to age $\mathrm{x}$ ) and the population parameters were calculated according to Ebrahimi et al. (2013). The intrinsic rate of increase $(r)$ was calculated on the basis of the Eule-Lotka equation as:

$=1($ Goodman, 1982) .

Net reproductive rate $\left(R_{0}\right)$ was measured as: 
The finite rate of increase $(\lambda)$ was equivalent to $e^{r m}$. The mean generation time (T) was defined as the time that the population could increase to $R_{0}$-fold of its population size as it approaches the stable stage distribution and was calculated as:

Finally, the means, standard errors and variances of the population parameters were estimated via the bootstrap technique (Efron \& Tibshirani, 1994), which is included in the Twosex-MS Chart programme (Chi, 1988). Sigmaplot 12.5 was used to create the graphs in this study (Ma et al., 2017).

\section{Statistical analysis}

The data of developmental time under constant temperature regimens $(18,21,24$, $27,30,33$ and $36^{\circ} \mathrm{C}$ ) were used to establish the temperature-developmental rate regression equation. The effective accumulative temperature $(K)$ and the developmental threshold temperature $(C)$ were calculated as follows:

(Liu et al., 2014)

(Liu et al., 2014)

where $T$ indicates the constant temperature of each treatment $(18,21,24,27,30,33$ or $\left.36^{\circ} \mathrm{C}\right), V$ represents developmental rate that was calculated as: $1 /$ development time, and $n$ is the number of the temperature treatments. The survival rates of larvae produced during the individual reproductive period were calculated as: survival rate of larva $=$ Number of pupa $/$ Number of total parasitized larvae $\times 100 \%$. The survival rate of pupa was calculated as: survival rate of pupa $=$ Number of emerging adult / Number of pupa $\times 100 \%$. The survival rates of immatures were calculated as: survival rate of immature $=$ Number of emerging adult / Number of total parasitized larvae $\times 100 \%$ (Li et al., 2006).

All data analyses were performed using SPSS 22.0 for Windows (IBM SPSS Inc. ${ }^{\circledR}$, Chicago, Illinois, version 22.0) and were checked for normality and homoscedasticity (Qiu et al., 2012). When the data did not fit a normal distribution, the parameters, such as progeny female ratio, survival rate of larva, survival rate of pupa and survival rate of immature, were arcsine transformed. If some parameters were not normal even after the transformation tests, the nonparametric Kruskal-Wallis test $(P<0.05)$ was performed then. The effects of different temperatures between each treatment was analysed by one-way analysis of variance using SPSS software, and means were compared by Turkey's multiple range test $(\alpha=0.01)$. Nonlinear regression was used to analyse the rate of development with temperature (using SPSS 22.0), and Sigma plot 12.5 was used to create the graphs (Chen et al., 2017a). 


\section{Results}

\section{Discussion}

Here, we found that M. similis can develop within the temperature range of 18 $33^{\circ} \mathrm{C}$ and that, within this range, their development parameters, longevity and fecundity increased with increasing temperature up to a threshold of $30^{\circ} \mathrm{C} . M$. similis were best adapted to the temperatures 27 and $30^{\circ} \mathrm{C}$, at which the parasitoid developed more rapidly, and the adults had a higher fecundity than at other temperatures, and beyond this temperature, the fecundity and longevity of $M$. similis rapidly fell. In addition, adult longevity, progeny female ratio, net reproductive rate $\left(R_{0}\right)$, intrinsic rate of natural increase $\left(r_{m}\right)$ and finite rate of increase $(\lambda)$ reached the peak at 27 and $30{ }^{\circ} \mathrm{C}$. Therefore, we conclude that the range $27-30^{\circ} \mathrm{C}$ is optimal for the development of M. similis.

Environmental factors are critical for the establishment and range expansion of parasitoids (Daane et al., 2004), and this is particularly important in their use for biological control. Temperatures outside the optimal range may slow parasitoid growth or, ultimately, halt parasitoid development (Edwin et al., 2016). In this study, $M$. similis can complete development at between 18 and $33{ }^{\circ} \mathrm{C}$, but only a few parasitoids emerged at $36{ }^{\circ} \mathrm{C}$. This result is similar to Liu et al. (2014), who demonstrated that the parasitoid Tetrastichus brontispae could not complete development in Brontispa longissimi larvae below $16{ }^{\circ} \mathrm{C}$ or above $32^{\circ} \mathrm{C}$. Temperature is a critical abiotic factor that influences the dynamics of arthropods and the parasitic wasps that develop in them (Jamshidnia \& Sadeghi, 2014). In our study, when the temperature increased (within the range $18-33^{\circ} \mathrm{C}$ ), the average adult longevity fell. This fall in longevity is in line with the results found for Aphidius erviwcrc developing in Aphis pomi larvae at $15-30{ }^{\circ} \mathrm{C}$ (Malina \& Praslicka, 2008).

Life table analyses are good methods for estimating parasitoid population development and its dynamics (Qiu et al., 2012). In this paper, the net reproductive rate $\left(R_{0}\right)$, the intrinsic rate of increase $\left(r_{m}\right)$ and finite rate of increase $(\lambda)$ of $M$. similis were the highest at 27 and $30{ }^{\circ} \mathrm{C}$, and those were significantly higher than other temperature treatments. These results indicate that the optimum developmental temperature of $M$. similis is $27-30{ }^{\circ} \mathrm{C}$, and this further suggests that $M$. similis has the potential to control S. litura, S. exigua and A. ipsilon because these pests are commonly found in this temperature range in southeast Asia. (Shepard \& Barrion, 1998; Shad et al., 2012; Ranjith et al., 2015). In the field, S. litura has an explosive population growth with overlapping generations, and a release of wasps into the field during the early growth of the pest population can contribute to slowing the rate of pest population growth and can make a valid 
contribution to an overall IPM strategy for this important pest. It is unclear how well the performance of the parasitoid would compare with the population growth of the pests concerned under higher field temperatures, but the results of this study suggest that the parasitoid would be impaired by the higher temperatures.

Estimating the developmental threshold temperature and effective accumulated temperature of a parasitoid provides important information for estimating the generation time of the insect in the field and selecting suitable conditions for the release of a parasitoid (Pratissoli et al., 2005). Here, the developmental threshold temperature and effective accumulated temperature of $M$. similis reared on larvae of $S$. litura were $9.96{ }^{\circ} \mathrm{C}$ and $231.14 \mathrm{DDs}$, respectively. The results of our study indicate that $M$. similis is unsuited for development at relatively low temperatures found in temperate and subtropical regions, although this parasitoid can enter dormancy or diapause (Qiu et al., 2012). Using the developmental threshold temperature established here and meteorological data, we can predict the number of generations of M. similis in a year. For example, in Changsha Southern China, where these studies were conducted, M. similis may have about 15-16 generations per year (Huang et al., 2011). The developmental threshold temperature of egg, larvae and pre-oviposition of S. litura are 8.2, 10.0 and 10.8, respectively (Rao et al., 1989). According to our study, the developmental threshold temperature of M. similis from egg to adult was the same as S. litura, indicating that $M$. similis can be potentially utilized in controlling $S$. litura in the same environment. In this paper, the number of generations of $M$. similis per year was calculated based on the constant laboratory temperatures. However, the actual temperature is extremely unstable in the field, which leads to the parasitoid's developmental rate varying from that found in the laboratory (Chi \& Su, 2006). Consequently, the lower thermal thresholds and effective accumulated temperature of M. similis in the field will require further study.

We found that $M$. similis can successfully complete their life cycle over a temperature range of $18-33{ }^{\circ} \mathrm{C}$ and that $27-30{ }^{\circ} \mathrm{C}$ was the optimal temperature for $M$. similis parasitoid larvae. The number of parasitoid generations per year has a significant impact on the control of populations of $S$. litura. This is important for biological control of the pest, but it is also important information for mass-rearing and releasing of $M$. similis in the field. Further studies are also required to investigate how periods of extreme high temperatures impact these organisms under field conditions.

\section{Acknowledgements}

The authors thank Miss Ze-Jun Tang, Miss Lin Li, Miss Yong-Xi Li, Miss Si-Yuan Wang and Mr. Ling-Cong Deng for their kind help in the experiments. This study was supported by the National Natural Science Foundation of China (31872027) and Changsha Science and Technology Project (kq1901033) and Double First- 
class Construction Project of Hunan Agricultural University. We declare that we do not have any commercial or associative interest that represents a conflict of interest in connection with the work submitted. 